\title{
Tsallis Entropies of Superposition States of Two Photon-Subtracted SU(1, 1) Coherent States and Entanglement Transfer to Qubits
}

\author{
MAi-Lin LianG*, Bing YuAn And JiA-NAN Zhang \\ Physics Department, School of Science, Tianjin University, Tianjin 300072, China
}

(Received September 18, 2008; in final form November 2, 2009)

\begin{abstract}
Entropy properties of the superposed photon-subtracted two-mode coherent states are quite different from that of the single state. Tsallis entropies of the superposed states have extreme values, while that of the single state change monotonously. Further, it is found that the positions of the maxima of the entropies are independent of the entropic index. Around the maxima of the entropies, the entanglement transfer from the superposed states to two initially separable qubits is nearly complete.
\end{abstract}

PACS numbers: 42.50.Dv, 03.65.Ud, 05.70.-a

\section{Introduction}

As an important type of two-mode continuous variable correlated states, the $\mathrm{SU}(1,1)$ coherent state $[1,2]$ has attracted a lot of investigations including its squeezing and statistical properties [3], superpositions [4], entanglement properties and entanglement transfer to qubits [5-8]. In quantum information network, a continuous variable system easily propagates entanglement while a qubit system is easy to manipulate. Therefore, the studies of entanglement transfer from a continuous variable system to a qubit system [6-12] are primary important due to their practical applications. The former investigations show that the entanglement of the qubits seems irrelevant of the magnitude of the entanglement of the initial continuous variable field [6].

In this paper, we study the following superposition states of two photon-subtracted $\mathrm{SU}(1,1)$ coherent states

$$
\left|\psi_{m}(\xi, p)\right\rangle=(a b)^{m}\left(|\xi, p\rangle+\delta \mathrm{e}^{\mathrm{i} \varphi}\left|\mathrm{e}^{\mathrm{i} \beta} \xi, p\right\rangle\right) / \sqrt{N}
$$

where $|\xi, p\rangle$ is the $\mathrm{SU}(1,1)$ coherent state $[1,2], a, b$ are the annihilation operators of the field modes, $\delta, \varphi, \beta$ are all real parameters, $m$ is an integer, $N$ is a normalization factor (to be given below). States (1) are the superpositions of the two photon-subtracted $\mathrm{SU}(1,1)$ coherent states $(a b)^{m}|\xi, p\rangle$ and $(a b)^{m}\left|\mathrm{e}^{\mathrm{i} \beta} \xi, p\right\rangle$. Of course, states (1) can also be named the photon-subtracted superposition states of two $\mathrm{SU}(1,1)$ coherent states $|\xi, p\rangle$ and $\left|\mathrm{e}^{\mathrm{i} \beta} \xi, p\right\rangle$. In the case of $m=0$, the states (1) are the superpositions of two $\mathrm{SU}(1,1)$ coherent states differing

* corresponding author; e-mail: mailinliang@yahoo.com.cn; in phase by $\beta$. The angle $0 \leq \varphi \leq 2 \pi$ affects the interference of the two coherent states. When $\delta=1, \beta=\pi$ and $\varphi=0, \pi$, the state $\left|\psi_{0}(\xi, p)\right\rangle$ becomes the even and odd $\mathrm{SU}(1,1)$ coherent states. When $\delta \neq 1$, the superposition coefficients are different in magnitude.

Using the scheme proposed in [4], the state $\left|\psi_{0}(\xi, p)\right\rangle$ can be generated by assuming that initially the two-level atom is in the state $\left(|g\rangle+\delta \mathrm{e}^{\mathrm{i} \varphi}|e\rangle\right) / \sqrt{1+\delta^{2}}$ and the field in the state $|\xi, p\rangle$. To generate $\left|\psi_{m}(\xi, p)\right\rangle$, we inject a two-level atom in the ground state into a cavity prepared in the state $\left|\psi_{0}(\xi, p)\right\rangle$. In the case of on-resonant interaction and a very short interaction time [13], the wave function of the whole system becomes

$$
\begin{aligned}
& |\psi(t)\rangle=\exp \left(-\mathrm{i} g t\left(a^{+} b^{+}|g\rangle\langle e|+a b| e\rangle\langle g|\right)\right)\left|\psi_{0}\right\rangle|g\rangle \\
& \quad \approx\left[1-\mathrm{i} g t\left(a^{+} b^{+}|g\rangle\langle e|+a b| e\rangle\langle g|\right)\right]\left|\psi_{0}\right\rangle|g\rangle \\
& \quad=\left|\psi_{0}\right\rangle|g\rangle-\mathrm{i} g t a b\left|\psi_{0}\right\rangle|e\rangle .
\end{aligned}
$$

Then, the state of the atom is detected. If the atom is found to be in the excited state, the field will be in the state $a b\left|\psi_{0}(\xi, p)\right\rangle$, which is a photon-subtracted superposition state. Repeating the process gives the state $\left|\psi_{m}(\xi, p)\right\rangle$. For $p=0$, the state $|\xi, p\rangle$ becomes the two-mode squeezed vacuum state [6] and $\left|\psi_{m}(\xi, p)\right\rangle$ is the superposed photon-subtracted two-mode squeezed vacuum states. Our calculations mainly focus on this case.

For pure states, entanglement can be measured by the linear entropy $[5,14]$ or the entropy of the reduced density matrix $[15,16]$

$$
S_{\mathrm{R}}=-\operatorname{Tr}\left(\rho_{\mathrm{A}} \log _{2} \rho_{\mathrm{A}}\right),
$$

where $\rho_{\mathrm{A}}=\operatorname{Tr}_{\mathrm{B}} \rho_{\mathrm{AB}}$ is the reduced density matrix with $\rho_{\mathrm{AB}}$ being the density matrix of the system having two subsystems A and B. The entropy of the reduced density 
matrix and the linear entropy are special cases of the Tsallis entropies [17-20]:

$$
S_{q}=\frac{1}{q-1}\left(1-\operatorname{Tr} \rho_{\mathrm{A}}^{q}\right),
$$

where $q$ is the entropic index. For $q=1,2$, the Tsallis entropies (3) reduce to the entropy of the reduced density matrix (3) and the linear entropy, respectively. Therefore, as a unified description of entanglement, we may calculate the Tsallis entropies. The Tsallis entropies have been used to describe many complex physical phenomena such as hydrodynamic turbulence [21], fluxes of cosmic rays [22], astrophysics [23], etc. There are also many discussions about using the non-extensive statistical mechanics to describe quantum entanglement [24-30].

In this paper, we study the Tsallis entropies of the Schrödinger cat states (1) and the entanglement transfer to two initially separable qubits. Numerical calculations show that when $\delta>0$ and $\varphi, \beta$ are both around $\pi$, the Tsallis entropies of (1) have extreme values and the positions of the maxima are independent of the entropic index $q$. Furthermore, at the maxima of the Tsallis entropies, the entanglement of the two qubits takes maximum values. Our arrangements are as follows. The next section is the Tsallis entropies of the superposition states (1). The third section is the entanglement transfer from the superposition states to qubits. The final section is the summary.

\section{Entanglement of the superposition states}

For $p=0$, the $\mathrm{SU}(1,1)$ coherent state $[1,2]$ reduces to the two-mode squeezed vacuum state

$$
|\xi, 0\rangle=A_{0} \sum_{n=0}^{\infty} \xi^{n}|n, n\rangle,
$$

where $A_{0}=\left(1-|\xi|^{2}\right)^{1 / 2}$ is the normalization constant and $|\xi| \equiv r$ is less than one. In terms of Fock states, the wave function (1) can be rewritten as

$$
\begin{aligned}
|\psi\rangle & =\sum_{n=0}^{\infty} C_{n}|n, n\rangle, \\
C_{n} & =\frac{A_{0}}{\sqrt{N}}\left(1+\delta \mathrm{e}^{\mathrm{i} \varphi+\mathrm{i} \beta(n+m)}\right) \prod_{k=1}^{m}(n+k) \xi^{n+m} .
\end{aligned}
$$

The normalization factor is found to be

$$
\begin{aligned}
N & =A_{0}^{2} \sum_{n=0}^{\infty}\left[1+\delta^{2}+2 \delta \cos (\varphi+\beta n+\beta m)\right] \\
& \times\left[|\xi|^{n+m} \prod_{k=1}^{m}(n+k)\right]^{2} .
\end{aligned}
$$

The corresponding Tsallis entropies are

$$
S_{\mathrm{f}}=\frac{1}{q-1}\left(1-\sum_{n=0}^{\infty} \rho_{n}^{q}\right), \quad \rho_{n}=\left|C_{n}^{2}\right|,
$$

where the subscript " $\mathrm{f}$ " means field.

At first we point out that Tsallis entropies of the photon-subtracted $\mathrm{SU}(1,1)$ coherent state $(a b)^{m}|\xi, p\rangle$ change monotonously, which is similar to that of a sin- gle two-mode coherent state $[5,6]$. Without loss of generality, we set $\delta>0$ for the superposition states (1). Numerical calculations show that for odd $m$, the Tsallis entropies change monotonously, too. However, for even $m$, the Tsallis entropies have extreme values when $\varphi, \beta$ are around $\pi$ as seen from Fig. 1 for $\delta=1.30, m=4$ and $\varphi=\beta=\pi$. One may notice that the position of the maxima is independent of the entropic index $q$. In Fig. 1, the maxima appear at $r=0.026$ for different $q$. For $\varphi=0.95 \pi, \beta=\pi$, the maxima are found to be at $r=0.031$. At the maxima of the Tsallis entropies, the entropy of the reduced density matrix is near one. We see that for different entropic index $q$, the values of the Tsallis entropies are different, but the behaviors are similar. That is to say, using the Tsallis entropies to measure the entanglement is appropriate.

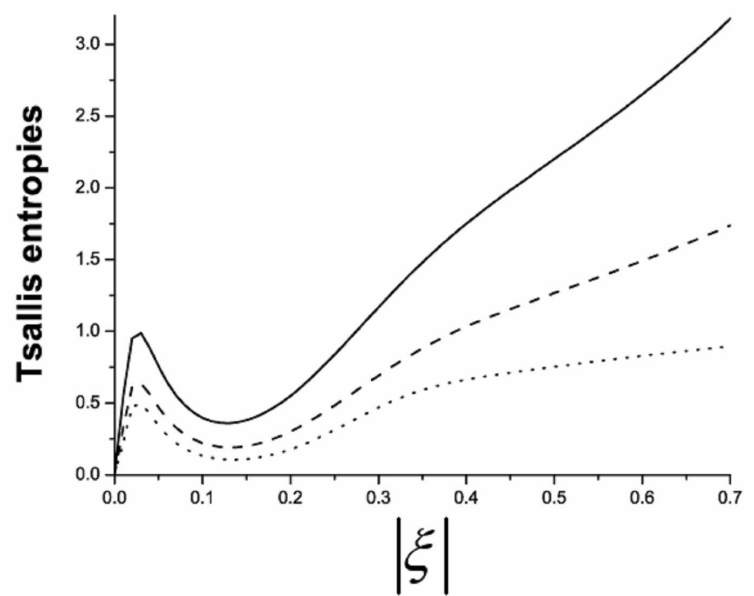

Fig. 1. Tsallis entropies versus $|\xi|$ for $p=0, m=4$, $\delta=1.30, \varphi=\pi, \beta=\pi$. The three curves (from top to bottom) correspond to $q=1,1.2,1.95$, respectively.

Numerical calculations also show that the extreme value of the Tsallis entropies vanishes when $\varphi=\beta=\pi$ and $\delta \cong 1$. This conclusion is true for arbitrary $m$. If $\varphi$ or $\beta$ is not $\pi$, there will be extreme values for the whole range $\delta$, roughly speaking from 0.4 to 2.5 .

To understand the properties of the Tsallis entropies qualitatively, for odd $m$ we rewrite the wave function (6) as (To be mathematically simple, here we take the case $\varphi=\beta=\pi$ as an example.)

$$
\begin{aligned}
& |\psi\rangle=\frac{\xi^{m} A_{0}}{\sqrt{N}} \sum_{n=0}^{\infty} \xi^{2 n}\left[(1+\delta) \prod_{k=1}^{m}(2 n+k)|2 n, 2 n\rangle\right. \\
& \left.+\xi(1-\delta) \prod_{k=1}^{m}(2 n+k+1)|2 n+1,2 n+1\rangle\right] .
\end{aligned}
$$

For even $m$, the state (6) can be cast into

$$
\begin{aligned}
& |\psi\rangle=\frac{\xi^{m} A_{0}}{\sqrt{N}} \sum_{n=0}^{\infty} \xi^{2 n}\left[(1-\delta) \prod_{k=1}^{m}(2 n+k)|2 n, 2 n\rangle\right. \\
& \left.+\xi(1+\delta) \prod_{k=1}^{m}(2 n+k+1)|2 n+1,2 n+1\rangle\right] .
\end{aligned}
$$


The terms within the square bracket in (9) or (10) have the form of the following Bell-like entangled number states:

$$
|\psi\rangle=\sqrt{x}|n, n\rangle+\mathrm{e}^{\mathrm{i} \theta} \sqrt{1-x}|n+1, n+1\rangle,
$$

where $1 \geq x \geq 0$ and $\theta$ is real. The Tsallis entropies of the entangled number states are

$$
S_{q}=\frac{1}{q-1}\left[1-x^{q}-(1-x)^{q}\right]
$$

which is zero for $x=0,1$ and has a maximum at $x=1 / 2$. The position $x=1 / 2$ of the maximum entropy is independent of the entropic index $q$. At the maximum, the absolute values of the coefficients before the two Fock states $|n, n\rangle$ and $|n+1, n+1\rangle$ are equal: $\sqrt{x}=\sqrt{1-x}$. Applying this condition to (9), we have

$$
|\xi|=\frac{1+\delta}{|1-\delta|} \frac{\prod_{k=1}^{m}(2 n+k)}{\prod_{k=1}^{m}(2 n+k+1)},
$$

which is greater than one for $2.5>\delta>0.4$ and arbitrary $n$. For the state (10), we have

$$
|\xi|=\frac{|1-\delta|}{(1+\delta)} \frac{\prod_{k=1}^{m}(2 n+k)}{\prod_{k=1}^{m}(2 n+k+1)},
$$

which is less than one. As $|\xi|$ is less than one for the states (1) or (6), it is understandable that there are no maxima for odd $m$ and exist maxima for even $m$.

In the next section, we will see that the maxima of the Tsallis entropies are very important to the entanglement transfer from the superposition states (1) to initially separable qubits.

\section{Entanglement transfer from the superposition states to qubits}

In interaction picture, the qubit-field interaction Hamiltonian is

$$
H_{a I}=\left(\Delta_{a} / 2\right) \sigma_{z}+g_{a}\left(|e\rangle\left\langle g\left|a+a^{+}\right| g\right\rangle|e\rangle\right),
$$

where $|g\rangle$ and $|e\rangle$ denote, respectively, the ground and excited states of the qubit, $\sigma_{z}$ is the Pauli matrix, $\Delta_{a}$ denotes the detuning between the field mode frequency and the corresponding qubit transition frequency. Replacing $a$ by $b$ in (15), we have the interaction Hamiltonian of mode $b$ with the other qubit. The time evolution operator of the whole system is then

$$
U(t)=u_{a}(t) \otimes u_{b}(t)
$$

where

$$
\begin{aligned}
& u_{a}(t)=\exp \left(-\mathrm{i} H_{a I} t\right)= \\
& {\left[\begin{array}{cr}
\cos \left(A_{a} t\right)-\mathrm{i} \frac{\Delta_{a} \sin \left(A_{a} t\right)}{2 A_{a}} & -\mathrm{i} g_{a} a \frac{\sin \left(B_{a} t\right)}{B_{a}} \\
-\mathrm{i} g_{a} a^{+\frac{\sin \left(A_{a} t\right)}{A_{a}}} \cos \left(B_{a} t\right)+\mathrm{i} \frac{\Delta_{a} \sin \left(B_{a} t\right)}{2 B_{a}}
\end{array}\right]} \\
& A_{a}=\sqrt{\left(\frac{\Delta_{a}}{2}\right)^{2}+g_{a}^{2} a a^{+}} \\
& B_{a}=\sqrt{\left(\frac{\Delta_{a}}{2}\right)^{2}+g_{a}^{2} a^{+} a}
\end{aligned}
$$

The operator $u_{b}(t)$ is obtained by replacing $a$ with $b$ in (17). Assuming that the initial state of the field is
(1) and the two qubits are both at the ground states, the wave function of the whole system at any time is

$$
|\psi(t)\rangle=U(t)|\psi\rangle|g g\rangle \text {. }
$$

The reduced density matrix for the two qubits is obtained by tracing out the fields as

$$
\rho_{q u}(t)=\operatorname{Tr}_{a b}|\psi(t)\rangle\langle\psi(t)| \text {. }
$$

After some calculations, the reduced density operator (19) for the two qubits is derived

$$
\rho_{q u}(t)=\left[\begin{array}{cccc}
A(t) & 0 & 0 & E(t) \\
0 & B(t) & 0 & 0 \\
0 & 0 & F(t) & 0 \\
E^{*}(t) & 0 & 0 & D(t)
\end{array}\right],
$$

where the matrix basis is chosen as $\{|e e\rangle,|e g\rangle,|g e\rangle,|g g\rangle\}$. The coefficients in (20) are

$$
\begin{aligned}
& A(t)=\sum_{n=0}^{\infty} \frac{n^{2}}{n_{a} n_{b}}\left|C_{n}^{2}\right| \sin ^{2}\left(g_{a} t \sqrt{n_{a}}\right) \\
& \times \sin ^{2}\left(g_{b} t \sqrt{n_{b}}\right), \\
& B(t)=\sum_{n=0}^{\infty} \frac{n}{n_{a}}\left|C_{n}^{2}\right| \sin ^{2}\left(g_{a} t \sqrt{n_{a}}\right) \\
& \times\left[\cos ^{2}\left(g_{b} t \sqrt{n_{b}}\right)+\frac{\Delta_{b}^{2}}{4 n_{b} g_{b}^{2}} \sin ^{2}\left(g_{b} t \sqrt{n_{b}}\right)\right], \\
& F(t)=\sum_{n=0}^{\infty}\left\{\frac { n } { n _ { b } } | C _ { n } ^ { 2 } | \left[\cos ^{2}\left(g_{a} t \sqrt{n_{a}}\right)\right.\right. \\
& \left.\left.+\frac{\Delta_{a}^{2}}{4 n_{a} g_{a}^{2}} \sin ^{2}\left(g_{a} t \sqrt{n_{a}}\right)\right] \sin ^{2}\left(g_{b} t \sqrt{n_{b}}\right)\right\}, \\
& D(t)=\sum_{n=0}^{\infty}\left\{| C _ { n } ^ { 2 } | \left[\cos ^{2}\left(g_{a} t \sqrt{n_{a}}\right)\right.\right. \\
& \times \\
& \times\left[\Delta_{a}^{2} \sin ^{2}\left(g_{a} t \sqrt{n_{a}}\right)\right] \\
& \left.\times\left(\cos _{a} g_{a}^{2} t \sqrt{n_{a}}\right)-\mathrm{i} \frac{\Delta_{a}}{2 g_{a} \sqrt{n_{a}}} \sin \left(g_{a} t \sqrt{n_{a}}\right)\right] \\
& \left.\times\left[\cos ^{2}\left(g_{b} t \sqrt{n_{b}}\right)+\frac{\Delta_{b}^{2}}{4 n_{b} g_{b}^{2}} \sin ^{2}\left(g_{b} t \sqrt{n_{b}}\right)\right]\right\}, \\
& \left.\quad t)=-\sum_{n=0}^{\infty}\left\{\frac{n+1}{2 g_{b} \sqrt{n_{b}}} \sin \left(g_{b} t \sqrt{n_{b}}\right)\right]\right\},
\end{aligned}
$$

where

$$
n_{a}=n+\left(\frac{\Delta_{a}}{2 g_{a}}\right)^{2}, \quad n_{b}=n+\left(\frac{\Delta_{b}}{2 g_{b}}\right)^{2} .
$$

Using (20) and (21), we can study the entanglement between the two qubits.

There are different ways to quantify the entanglement of the two qubits, such as the concurrence [31, 32] and the negativity of the partial transposition of the reduced density matrix $[33,34]$. The concurrence is defined as 


$$
C=\max \left\{0,2 \max \left[\lambda_{i}\right]-\sum_{i}^{4} \lambda_{i}\right\}
$$

with $\lambda_{i}$ being the square roots of the eigenvalues of the matrix $R=\rho_{q u} S \rho_{q u}^{*} S$, where $S=\sigma^{y} \otimes \sigma^{y}$ and $*$ stands for the complex conjugate. For the present system, the square roots of the eigenvalues of the matrix $R$ are found to be

$$
\begin{aligned}
& \lambda_{1}=|\sqrt{A D}-| E||, \quad \lambda_{2}=\sqrt{A D}+|E|, \\
& \lambda_{3}=\lambda_{4}=\sqrt{B F} .
\end{aligned}
$$

The entanglement measure based on the negativity of the partially transposed density matrix is $\varepsilon=-2 \sum_{i} \lambda_{i}^{-}$, where $\lambda_{i}^{-}$are negative eigenvalues of the partially transposed density matrix $[33,34]$. In our case, we have

$$
\varepsilon=\sqrt{(B-F)^{2}+4|E|^{2}}-B-F .
$$

Numerical calculations show that the concurrence and the negativity are equal to each other for $p=0$.

For $g_{a}=g_{b}=g$, the concurrence reaches its maximum value at the scaled times $g t=(0.5+k) \pi$ ( $k$ is an integer). For example, when $\delta=1.30, m=4, g_{a}=g_{b}=1$, $\Delta_{a}=\Delta_{b}=0$ and $\varphi=0.95 \pi, \beta=\pi$, the maximum of the Tsallis entropies appears at $r=0.031$ and the concurrence $C$ is $0.9995,0.9996$ and 0.9995 at the times $g t=\pi / 2,3 \pi / 2,5 \pi / 2$, respectively. At these times, the two qubits are almost maximally entangled. If $|\xi|$ deviates from 0.031 , the concurrence decreases obviously as shown in Fig. 2 for $g t=3 \pi / 2$.

From Fig. 1, one can see that the Tsallis entropies become larger and larger when $|\xi|$ is greater than 0.3 . However, the entanglement of the two qubits does not increase with the increasing $|\xi|$, which agrees with the former calculations using the single two-mode coherent states [6]. Actually, for larger $|\xi|$, the concurrence becomes very small. For example, the largest possible concurrence is about 0.1 for $|\xi|=0.6$. The concurrence tends to zero when $|\xi|$ is greater than 0.7 .

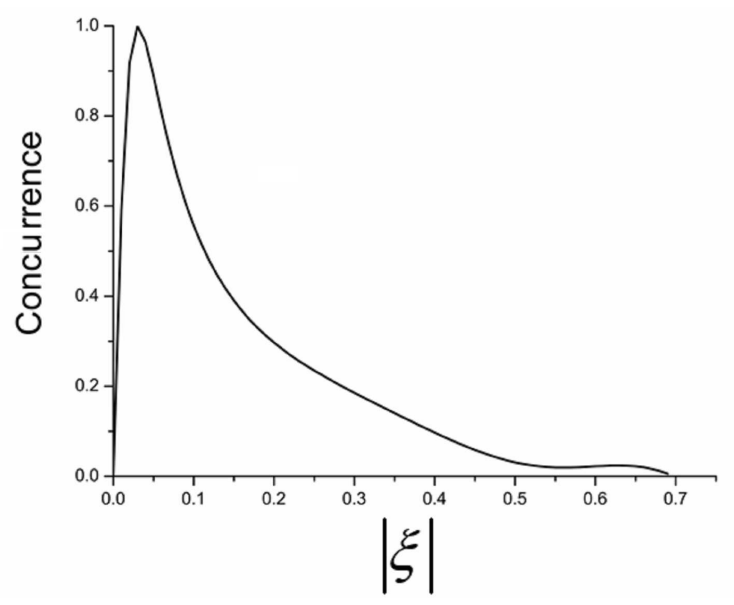

Fig. 2. Concurrence versus $|\xi|$.
The concurrence and negativity corresponding to other maxima of the field (1) have similar behaviors. To understand the above effective entanglement transfer at the maxima of the Tsallis entropies, it may be helpful to consider the case that the initial field is the entangled number states (11). For the entangled number states (11), the concurrence and negativity are both $C=\varepsilon=$ $2 \sqrt{x(1-x)}$. Under the conditions that $\Delta_{a}=\Delta_{b}=0$, $g_{a}=g_{b}=\mathrm{g}$ and the qubits are initially at the ground states, the concurrence and the negativity of the qubits are found to be equal

$$
\begin{aligned}
C & =\varepsilon=2 \sqrt{x(1-x)} \cos ^{2}(n g t) \sin ^{2}((n+1) g t) \\
& -2 x \sin ^{2}(n g t) \cos ^{2}(n g t) \\
& -2(1-x) \sin ^{2}((n+1) g t) \cos ^{2}((n+1) g t) .
\end{aligned}
$$

For even $n$, the concurrence and negativity (26) are $2 \sqrt{x(1-x)}$ at the scaled times $g t=(0.5+k) \pi$. In another words, the entanglement of the field is completely transferred to the qubits. When $x=1 / 2$, the qubits are maximally entangled. For odd $n$, there is no such result.

Now we have a look at the atomic state. For $g_{a}=g_{b}$ and $\Delta_{a}=\Delta_{b}=0$, numerical calculations give $A \rightarrow$ $1 / 2, D \rightarrow 1 / 2, E \rightarrow-1 / 2, B \rightarrow 0$ and $F \rightarrow 0$ at the scaled times $g t=(0.5+k) \pi$. Meanwhile, by calculating the entropy $S_{q f}=-\operatorname{Tr}\left(\rho_{q u} \log _{2} \rho_{q u}\right)$, we know that the qubits and the fields are now disentangled. (The roots of the reduced density matrix $\rho_{q u}$ are $r_{1}=B \rightarrow 0, r_{2}=$ $F \rightarrow 0, r_{3,4}=\left[(A+D) \pm \sqrt{(A-D)^{2}+4|E|^{2}}\right] / 2 \rightarrow 1,0$, which results in $S_{q f} \rightarrow 0$ or the qubits and the fields are disentangled.) From these results, we can conclude that the state of the two qubits evolves into $(|e e\rangle-|g g\rangle) / \sqrt{2}$.

The detuning effects are also considered. It is shown that when $\left|\Delta_{j}\right| / g_{1,2} \leq 10 \%$, the qubits still keep highly entangled. For example, when $\delta=1.25, r=0.037$, $m=2, g_{a}=g_{b}=1, \Delta_{a}=0.1, \Delta_{b}=-0.1$ and $\varphi=\beta=\pi$, the concurrence $C$ is 0.9945 and 0.9940 at the scaled times $g t=3 \pi / 2,5 \pi / 2$, respectively.

When the two coupling constants are not the same, the concurrence and negativity tend to decrease. For $m=4, \Delta_{1}=\Delta_{2}=0, g_{b}=1, \delta=1.30, r=0.031$, $\varphi=0.95 \pi, \beta=\pi$ and $g_{a}=1.01,1.02,1.03,1.06$, 1.07 , the corresponding concurrences are, respectively, $0.9992,0.9988,0.9981,0.9945,0.9928$. When $\beta=1.03 \pi$ $(r=0.047), g_{1}=1.03,1.05$, the values of the concurrence are $0.9965,0.9920$. Calculations show that the concurrence and negativity are greater than 0.99 so long as $\left|g_{a}-g_{b}\right| / g_{a, b} \leq 6 \%$.

In case that the particle number difference $p$ is not zero, the concurrence and negativity become much smaller. For example, when $p=2, \delta=1.30, r=0.022, m=4$, $\Delta_{1}=\Delta_{2}=0, g_{a}=g_{b}=1$ and $\varphi=\beta=\pi$, the largest possible concurrence is 0.974 at $g t=4.53$.

From the above discussions, we see that when $m$ is even and $\varphi, \beta$ are around $\pi$, the concurrence and negativity of the two qubits tend to unit. In other words, the two qubits are nearly maximally entangled. 


\section{Summary}

We have studied the Tsallis entropies of the superposed photon-subtracted two-mode squeezed vacuum states and the entanglement transfer to two initially separable qubits. For odd $m$, there are no special results. But for even $m$, a new phenomenon appears: the Tsallis entropies can have extreme values. Further, the positions of the maxima are independent of the entropic index $q$. When the Tsallis entropies take the extreme values, the two qubits can be almost maximally entangled. The initial field and the qubit-field interaction Hamiltonian presented here can be realized experimentally. Hence, the results presented in this paper can be tested.

\section{References}

[1] C.C. Gerry, J. Opt. Soc. Am. B 8, 685 (1991).

[2] C.C. Gerry, R.E. Welch, J. Opt. Soc. Am. B 9, 290 (1992).

[3] L. Gilles, P.L. Knight, J. Mod. Opt. 39, 1411 (1992).

[4] C.C. Gerry, R. Grobe, J. Mod. Opt. 44, 41 (1997).

[5] A.S. Agarwal, A. Biswas, J. Opt. B 7, 350 (2005).

[6] W. Son, M.S. Kim, J. Lee, D. Ahn, J. Mod. Opt. 49, 1739 (2002).

[7] M. Paternostro, W. Son, M.S. Kim, G. Falci, G.M. Palma, Phys. Rev. A 70, 022320 (2004).

[8] J. Zou, J.G. Li, B. Shao, J. Li, Q. Shuli, Phys. Rev. A 73, 042319 (2006)

[9] J. Hald, J.L. Sorensen, C. Schori, E.S. Polzik, J. Mod. Opt. 47, 2599 (2000).

[10] M. Paternostro, W. Son, M.S. Kim, Phys. Rev. Lett. 92, 197901 (204).

[11] M. Paternostro, G. Falci, M.S. Kim, G.M. Palma, Phys. Rev. B 69, 214502 (2004).

[12] X.F. Qian, Y. Li, Y. Li, Z. Song, C.P. Sun, Phys. Rev. A 72, 062329 (2005).
[13] G.S. Agarwal, K. Tara, Phys. Rev. A 43, 492 (1991).

[14] D.A. Meyer, N.R. Wallach, J. Math. Phys. 43, 4273 (2002).

[15] M.B. Plenio, V. Vedral, Contemp. Phys. 39, 431 (1989).

[16] S.M. Barnett, S.J.D. Phoenix, Phys. Rev. A 40, 2204 (1989); S.M. Barnett, P.L. Knight, J. Opt. Soc. Am. B 2, 467 (1985).

[17] C. Tsallis, J. Stat. Phys. 52, 479 (1988).

[18] A.R. Plastino, A. Plastino, Phys. Lett. A 177, 177 (1993).

[19] C. Tsallis, S.V.F. Levy, A.M.C. Souza, R. Maynard, Phys. Rev. Lett. 75, 3589 (1995).

[20] N.G. de Almeida, Physica A 387, 2745 (2008).

[21] C. Beck, Phys. Rev. Lett. 87, 180601 (2001).

[22] C. Tsallis, J.C. Anjos, E.P. Borges, Phys. Lett. A 310, 372 (2003).

[23] C. Tsallis, D. Prato, A.R. Plastino, Astrophys. Space Sci. 290, 259 (2004).

[24] S. Abe, A.K. Rajagopal, Phys. Rev. A 60, 3461 (1999).

[25] S. Abe, A.K. Rajagopal, Physica A 283, 157 (2001).

[26] N. Canosa, R. Rossignoli, Phys. Rev. Lett. 88, 170401 (2002).

[27] J. Batle, A.R. Plastino, M. Casas, A. Plastino, J. Phys. A 35, 10311 (2002).

[28] S. Abe, Physica A 306, 316 (2002).

[29] R. Prabhu, A.R.U. Devi, G. Padmanabha, Phys. Rev. A 76, 042337 (2007).

[30] G. Wilk, Z. Wlodarczyk, Physica A 376, 279 (2007).

[31] S. Hill, W.K. Wootters, Phys. Rev. Lett. 78, 5022 (1997).

[32] W.K. Wootters, Phys. Rev. Lett. 80, 2245 (1998).

[33] A. Peres, Phys. Rev. Lett. 77, 1413 (1996).

[34] M. Horodecki, P. Horodecki, R. Horodecki, Phys. Lett. A 223, 1 (1996). 